\title{
Synthetic lethality interaction between Aurora kinases and CHEK1 inhibitors in ovarian cancer
}

Ana Alcaraz-Sanabria ${ }^{1}$, Cristina Nieto-Jiménez ${ }^{1}$, Verónica Corrales-Sánchez ${ }^{1}$, Leticia Serrano-Oviedo ${ }^{1}$, Fernando Andrés-Pretel', Juan Carlos Montero', Miguel Burgos ${ }^{3}$, Juan Llopis ${ }^{3}$, Eva María Galán-Moya ${ }^{3}$, Atanasio Pandiella ${ }^{2}$ and Alberto Ocaña ${ }^{1}$

from the

${ }^{1}$ Translational Research Unit, Albacete University Hospital, Albacete, Spain;

${ }^{2}$ Cancer Research Center, CSIC-University of Salamanca, Salamanca, Spain. ${ }^{3}$ Translational Oncology Laboratory, Centro Regional de Investigaciones Biomédicas (CRIB), Universidad de Castilla La Mancha, Albacete, Spain.

Key words: ovarian cancer, synthetic lethality, Aurora kinase inhibitor, CHEK1 inhibitor.

Running Title: AURKA and CHEK1 inhibitors synergize in ovarian cancer

Correspondence:

Alberto Ocaña MD, PhD

Translational Research Unit

Calle Francisco Javier de Moya

Albacete University Hospital

02006 Albacete, Spain

Phone: +34 967597100 ext 37087

Email: albertoo@sescam.jccm.es

Financial information:

This work has been supported by Instituto de Salud Carlos III (PI16/01121),CIBERONC, ACEPAIN; Diputación de Albacete and CRIS Cancer Foundation (to A. Ocaña). Ministry of Economy and Competitiveness of Spain (BFU2015-71371-R), the Instituto de Salud Carlos III through the Spanish Cancer Centers Network Program (RD12/0036/0003) and CIBERONC, the scientific foundation of the AECC and the CRIS Foundation (to A. Pandiella). The work carried out in our laboratories receive support from the European Community through the regional development funding program (FEDER). J.C. Montero is a recipient of a Miguel Servet fellowship program (CP12/03073) and receive research support from the ISCIII (grants PI15/00684). E.M. GalanMoya is funded by the implementation research program of the UCLM (UCLM resolution date: 31/07/2014), with a contract for accessing the Spanish System of Science, Technology and Innovation-Secti (co-funded by the European Commission/FSE funds). 


\section{Abstract}

Ovarian cancer is characterized by frequent mutations at TP53. These tumors also harbor germline mutations at homologous recombination repair (HR) genes, so they rely on DNA-damage checkpoint proteins, like the Checkpoint kinase 1 (CHEK1) to induce G2 arrest. In our study, by using an in silico approach, we identified a synthetic lethality interaction between CHEK1 and mitotic Aurora Kinase A (AURKA) inhibitors. Gene expression analyses were used for the identification of relevant biological functions. OVCAR3, OVCAR8, IGROV1 and SKOV3 were used for proliferation studies. Alisertib was tested as AURKA inhibitor and LY2603618 as CHEK1 inhibitor. Analyses of cell cycle and intracellular mediators were performed by flow cytometry and Western blot. Impact on stem cell properties was evaluated by flow cytometry analysis of surface markers and sphere formation assays. Gene expression analyses followed by functional annotation identified a series of deregulated genes which belonged to cell cycle, including AURKA/B, TTK kinase and CHEK1. AURKA and CHEK1 were amplified in $8.7 \%$ and $3.9 \%$ of ovarian cancers, respectively. AURKA and CHEK1 inhibitors showed a synergistic interaction in different cellular models. Combination of Alisertib and LY2603618 triggered apoptosis, reduced the stem cell population and increased the effect of taxanes and platinum compounds. Finally, expression of AURKA and CHEK1 was linked with detrimental outcome in patients. Our data describes a synthetic lethality interaction between $\mathrm{CHEK} 1$ and AURKA inhibitors with potential translation to the clinical setting. 


\section{Introduction}

Identification of druggable vulnerabilities is a main goal in oncogenic diseases where available therapies can only slightly prolong survival [1, 2]. Even though new therapies can reduce and delay tumor growth, most cancers become resistant, with the appearance of new clones of cells that are insensitive to the inhibited mechanisms [3].

Advanced ovarian cancer represents an important clinical problem since limited benefit can be obtained from available therapies [4]. In this context, it is mandatory to identify druggable mechanisms that are responsible for the oncogenic phenotype and that can augment the effectiveness of existing therapies.

The classical treatment in ovarian cancer includes anti-mitotic agents like taxanes in combination with DNA damaging agents like platinum compounds [4]. Recently, new agents have been incorporated to the therapeutic armamentarium including anti-angiogenic compounds such as the antibody bevacizumab or agents targeting DNA repair mechanisms like PARP inhibitors, among others [4-6].

Several molecular alterations have been described in ovarian cancer including, uncontrolled regulation of mitosis, deficiencies in DNA damage repair mechanisms or activation of intracellular pathways involved in proliferation and survival $[7,8]$. In this context, some mitotic protein kinases have been identified as upregulated and linked to worse outcome in ovarian cancer, constituting potential therapeutic targets [9]. Drugs against mitotic kinases like Polo-like kinases (PLK), Never in mitosis (NIMA) or Aurora kinases (AURK) have been recently described [10]. Moreover, massive genomic studies have reported 
overexpression of some of these proteins in ovarian carcinoma [11]. Indeed, agents against some of these proteins, such as those targeting AURKs are currently in clinical development $[12,13]$.

Control of DNA damage is a key function associated with the initiation and maintenance of ovarian cancer [14]. Genetic studies have reported frequent mutations in TP53 in high grade serous ovarian carcinoma [11]. Moreover, germline mutations at the homologous recombination repair genes BRCA1 and BRCA2 predispose to ovarian cancer and are currently used to identify tumors susceptible to be treated with PARP inhibitors [5]. Both functions, cell cycle control and DNA repair, are mechanistically linked as cells that have a high proliferation rate acquire more genetic instability, and supervision of DNA lesions and repair becomes more difficult [14]. Among the different proteins involved in the regulation and identification of DNA damage, the Checkpoint kinase 1 (CHEK1) is a key member [15]. It mediates cell cycle arrest in response to DNA damage by integrating the signals from ATM and ATR [15]. In addition, some mitotic kinases like Aurora or PLK are involved in the regulation of the DNA damage response through the phosphorylation of cell cycle regulators $[16,17]$.

In this context, targeting of DNA repair mechanisms in combination with inhibition of key regulators of the mitotic process could be an exploitable path to treat ovarian cancer.

In this article by using an in silico approach we identify Aurora Kinases A and B (AURKA and AURKB) and CHEK1 as an upregulated family of genes in ovarian cancer. Together AURKA and CHEK1 were amplified in around $12 \%$ of ovarian tumors. We show that inhibition of AURKA synergized with the inhibition of the 
DNA repair regulator CHEK1. The combined inhibition induced cell cycle arrest, apoptosis and synergized with chemotherapies, having also an effect on populations of cells with stem cell properties. In addition, the concomitant expression of these genes was linked to poor clinical outcome.

Taking together the data contained in our article paves the way for future preclinical studies and exploratory early phase clinical trials using this combination.

\section{Material and Methods}

Transcriptomic analysis, functional annotation and outcome analysis

We used public transcriptomic mRNA data (GEO DataSet, accession number: GDS3592) from non-transformed isolated human epithelial ovarian cells and ovarian carcinoma cells to identify deregulated genes. Affymetrix CEL files were downloaded and analyzed with Transcriptome Analysis Console (TAC) Software, developed by Affimetrix. Normalization was performed using MAS5. Genes that were upregulated with a minimum of 4 fold change were selected. Functional annotation was performed with DAVID Bioinformatics Resources 6.7 and adjusted $p$-value $<0.05$ to select the enriched gene-sets. Data contained at oncomine (https://www.oncomine.org/resource/login.html) was used to confirm the upregulated genes. Copy number alterations including amplifications, deletions or mutations were evaluated using cBioportal (http://www. cbioportal.org) [18]. For the association of gene expression with clinical outcome in early stage ovarian cancer we used the Kaplan Meier (KM) Plotter Online Tool [19].

Cell Culture and drug compounds 
The immortal human keratinocyte cell line HACAT was growth in DMEM. Ovarian cancer cell lines were growth in RPMI (OVCAR3, IGROV1) and DMEM (SKOV3, OVCAR8) containing 10\% fetal bovine serum (FBS). OVCAR3 and OVCAR8 tumorspheres were grown in DMEM-F12 plus BSA (0.4\%), insulin $(5 \mu \mathrm{g} / \mathrm{ml})$, bFGF $(20 \mathrm{ng} / \mathrm{ml})$ and EGF $(20 \mathrm{ng} / \mathrm{ml})$. All media were supplemented with $100 \mathrm{U} / \mathrm{mL}$ penicillin, $100 \mu \mathrm{g} / \mathrm{mL}$ streptomycin and $2 \mathrm{mM} \mathrm{L}$-glutamine and cells were maintained at $37^{\circ} \mathrm{C}$ in a $5 \% \mathrm{CO}_{2}$ atmosphere. All cell lines used were provided by Drs J Losada and A Balmain (from the ATCC) in 2015. In addition, cells are analyzed annually by STR at the molecular biology unit at the Salamanca University Hospital.

Cell culture media and supplements were obtained from Sigma Aldrich (St. Louis, MO). LY2603618, Alisertib, AZ3146 and Docetaxel were obtained from Selleckchem (Munich, Germany). Carboplatin was purchased from Pfizer GEP, SL. Cells were Mycoplasma free at different evaluations.

MTT, clonogenic assays and matrigel embedded 3D cultures

Dose-response and synergy studies were assessed by MTT screening assay. Ovarian cancer cell lines were seeded in 48-multiwell plates (10.000 cells per well) and treated with the indicated compounds and doses for the indicated times. To determine cell proliferation, MTT was added to the wells for $1 \mathrm{~h}$ at $37^{\circ} \mathrm{C}$ (0.5 mg/ml). Then, MTT was solubilized with DMSO and absorbance was measured at $562 \mathrm{~nm}(555-690)$ in a multiwell plate reader (BMG labtech). Results were plotted as the mean values of three independent experiments. Interaction among drugs was calculated using Calcusyn Version 2.0 software (Biosoft, Ferguson, $\mathrm{MO}$ ) by determining combinational index $(\mathrm{CI})$ based on the algorithm reported by Chou and Talalay. Values $<1$, synergistic effect, values 
equal to 1 , indicate additive effect, and values $>1$ represent an antagonistic effect. For three-dimensional (3D) cell culture experiments OVCAR3 and OVCAR8 cells were seeded in a 48-multiwell plate (10.000 cells per well) containing an underlying layer of Matrigel, which was pre-incubated at $37^{\circ} \mathrm{C}$ during 30 minutes. The following day, cells were treated with Alisertib, LY2603618 or the combination of them. 3D colonies number and diameter were daily monitorized under a light microscope for 5 days. For clonogenic experiments, cells were treated with Alisertib, LY2603618 and the combination of them for 24 hours (500.000 cells per well in a 6 well plate). Then, cells were tripsinized, count and resuspended in complete growth medium to perform serial dilutions $1 / 10$. We selected dilutions 3 and 4 and seeded in triplicate in 6 multiwell plates during 10 days, when number of colonies was counted.

LDH cytotoxicity assays

For LDH cytotoxicity assay, OVCAR3 and OVCAR8 were seeded in a 48multiwell plate (10.000 cells per well) and, the following day, treated with Alisertib, LY2603618 and the combination of both drugs for 72 hours. Then, LDH activity was evaluated following manufactured instructions (Pierce LDH Cytotoxicity Assay Kit, ThermoFisher Scientific).

Flow cytometry analysis

For cell cycle experiments, OVCAR3, OVCAR8 and SKOV3 were seeded and 24h later synchronized with double thymidine block (2 mM). Briefly, cells were exposed to thymidine for $18 \mathrm{~h}$, and then, after recovering in thymidine free medium for 9h, a second exposure was performed for another $18 \mathrm{~h}$. Then, cells were washed and treated with Alisertib, LY2603618 or the combination of them for $24 \mathrm{~h}$. Non-treated cells were used as a control. 
Cells were collected and fixed with $70 \%$ cold ethanol during 30 minutes. Then, cells were washed twice and Propidium iodide/RNAse staining solution (Immunostep S.L., Salamanca, Spain) was added. Results were analyzed on FACSCanto II flow cytometer (BD Biosciences). Percentage of cells in each cell cycle phase was determined by plotting DNA content against cell number using the FACS Diva software.

For apoptosis and caspase assays, cells were plated and, 24h later, pre-treated with $50 \mu \mathrm{M}$ of the pan-caspase inhibitor Z-VAD-FMK, before adding Alisertib, LY2603618 or the combination for $72 \mathrm{~h}$. Treated cells were collected and stained in the dark with Annexin V-DT-634 (Immunostep S.L., Salamanca, Spain) and Propidium iodide at room temperature for 1 hour. Apoptotic cells were determined using a FACSCanto II flow cytometer (BD Biosciences). Then, early apoptotic and late apoptotic cells were used in cell death determinations. For detection of CD44 and CD133 proteins cells were tripsinized, centrifugated and cell pellets were resuspended and incubated with CD44 (10 $\mu \mathrm{L} /$ sample) and CD133 (10 $\mu \mathrm{L} /$ sample) antibodies at $4^{\circ} \mathrm{C}$ for $1 \mathrm{~h}$ before being examined using a FACSCanto II flow cytometer (BD Biosciences).

Caspase-3 activity assays

Ovarian cancer cell lines OVCAR3 and OVCAR8 were lysed in apoptosis lysis buffer (20 mM Tris, 140 mM NaCl, 10 mM EDTA, 10\% glycerol, 1\% NP40, pH 7.0) supplemented with protease inhibitors. Protein concentration was determined by de BCA assay (Pierce) and $50 \mu \mathrm{g}$ of cell lysates were placed in 96-well plates. Caspase reaction buffer (50 mM HEPES pH 7.4, 300 mM NaCl, $2 \mathrm{mM}$ EDTA, 0.2\% CHAPS, 20\% sucrose, $20 \mathrm{mM}$ DTT and $10 \mu \mathrm{M}$ fluorescently labelled caspase substrate Ac-IETD-AFC or Ac-DEVD-AFC) was added to each 
Author Manuscript Published OnlineFirst on August 28, 2017; DOI: 10.1158/1535-7163.MCT-17-0223

Author manuscripts have been peer reviewed and accepted for publication but have not yet been edited.

well containing cell lysates. The plate was incubated at $37^{\circ} \mathrm{C}$ for 1 hour and signals were measured at 400/505 $\mathrm{nm}$ in a fluorescent reader (BioTek). 


\section{Immunofluorescence microscopy}

Ovarian cancer cells OVCAR3 and OVCAR8 were cultured on glass coverslips, washed with PBS and fixed in $2 \%$ p-formaldehyde (PFA) for 30 minutes at room temperature, followed by a wash in PBS. Monolayers were quenched for 10 minutes with PBS with $50 \mathrm{nM} \mathrm{NH} \mathrm{Cl}_{2}$. Then, cells were permeabilized for 10 minutes with $0.1 \%$ Triton $\mathrm{X}-100$ in PBS, washed again and blocked with $0.2 \%$ BSA in PBS for 10 minutes. Monolayers were incubated with and subsequently incubated overnight at $4^{\circ} \mathrm{C}$ with anti- $\beta$-tubulin $(1: 250$, Santa Cruz Biotechnologies) and anti-Nucleoporin p62 (1:200, BD transduction laboratories) primary antibodies. Cells were washed three times (3x) in PBS and incubated with an anti-mouse Alexa Fluor 568 (1:1000) antibody for 60 minutes. Cells were again washed with PBS (3x) and DAPI (300 nM) was added for 10 min and washed twice with PBS before mounting. Fluorescence imaging of cells was performed using an epifluorescence inverted microscope (DMIRE-2, Leica) with a PlanApo 40x oil immersion objective. Images were obtained in a Zeiss LSM 710 confocal microscope with a Plan Apo 63x oil immersion objective.

OVCAR3 and OVCAR8, grown as tumorspheres (TS) for 1 week, were dropped on poly-lysine slides for $1 \mathrm{~min}$ and then fixed with $4 \%$ of PFA for 10 minutes at RT. TS were permeabilized for 5 minutes with $0.1 \%$ Triton $\mathrm{X}-100$ in PBS, washed and blocked with $2 \%$ BSA for 30 minutes. Then, cells were incubated for 1 hour with R-phycoerythrin (PE)-coupled CD44 (Inmunostep, Salamanca, Spain) or Sox-2 (Millipore, Bedford, MA, USA). Sox-2 incubated TS were then washed with PBS and incubated with an anti-rabbit Alexa Fluor 568 antibody for 60 minutes. TS were again washed with PBS before mounting with Fluoroshield 
(Sigma Aldrich, St. Louis, MO). Fluorescence imaging of TS was performed using confocal microscopy (Zeiss LSM 710) with a 63x oil immersion objective.

\section{Western Blotting}

OVCAR3 and OVCAR8 cells were treated with Alisertib, LY2603618 or the combination of both drugs at the indicated doses. For PARP and $\mathrm{pH} 2 \mathrm{AX}$ apoptotic proteins and pAURKA, AURKA, pAURKB, pAURKC, pCHEK1, CHEK1, Cyclin A, p27 and p21 proteins detection, cells were before synchronized with double thymidine block before cells were treated as described in the flow cytometry section. After drug treatment, cells were lysed in cold RIPA lysis buffer supplemented with protease and phosphatase inhibitors (Sigma Aldrich, St. Louis, MO). Then, protein concentration was determined using Pierce BCA (Bicinchoninic acid) protein assay kit (Thermo Fisher Scientific, Waltham, MA USA). $50 \mu \mathrm{g}$ of total protein was loaded in a SDS-PAGE electrophoresis system. Blots were blocked in Tris-buffered saline (TBS)-5\% milk and incubated overnight with the following primary human antibodies: The anti-Cyclin B, anti-Wee1, anti-PARP, anti-p21 and anti-GAPDH antibodies were purchased from Santa Cruz Biotechnology (Santa Cruz, CA, USA). The antipH3 antibody was from Millipore Corporation (Bedford, MA, USA). The antipH2AX and anti-pCDK1-Y15, anti-pAurora (A,B,C), anti-Aurora A, anti-pCHEK1, anti-CHEK1 and anti-p27 antibodies were from Cell Signalling Technologies (Beverly, MA, USA). The anti-cyclin A antibody was purchased from BD Biosciences (San Jose, CA, USA),

Horseradish peroxidase conjugates of anti-rabbit and anti-mouse immunoglobulin G (IgG) were from Bio-Rad Laboratories (Hercules, CA, USA). 
Protein bands were visualized by a luminal-based detection system with $p$ iodophenol enhancement.

Secondary Tumorspheres formation and MTS assays

OVCAR3 and OVCAR8 tumorspheres (TS) were mechanically dissociated by pipetting, counted and cultured in 6 well plates $\left(10^{5}\right.$ cells per well) in the presence of Alisertib, LY2603618 or a combination of both drugs. Cells were dissociated again 24 and 48h later. At day 3, TS counts were blindly performed on six random fields of view (FOV). TS/FOV was calculated from 3 independent experiments.

For MTS proliferation assays (MTS Cell Proliferation Assay Kit, Abcam), OVCAR3 and OVCAR8 TS were seeded in a 48-multiwell plate (10.000 cells per well) and treated with Alisertib, LY2603618, Docetaxel and Carboplatin for $72 \mathrm{~h}$. Then, MTS reagent was added to culture media for $1 \mathrm{~h}$, following supplier manufactured instructions, and the absorbance was measured at $490 \mathrm{~nm}$.

Statistical analysis

All experiments were performed at least three times. Student t-test was used to determine significant statistical differences. Two-ways Student's test was used for the statistical analyses $\left(p \leq 0.05^{*}, p \leq 0.01^{* *}, p \leq 0.001^{* * *}\right)$. 


\section{Results}

In silico transcriptomic analyses identify druggable kinases in ovarian cancer To identify molecular functions dysregulated in ovarian tumors, we performed transcriptomic analyses guided by functional genomics. We used a public dataset containing information from non-transformed isolated human epithelial ovarian cells and ovarian carcinoma cells (GEO DataSet accession number: GDS3592). Using a minimum fold change of four we select 2925 genes (Figure 1A). Functional analyses identified deregulation of relevant functions including cell differentiation, response to stress or cell cycle, among others (Figure 1A). Taking in consideration the relevant role of cell cycle mediators in cancer, we searched for druggable kinases contained within this function, identifying only four druggable genes: AURKA, AURKB, CHEK1 and TTK/MPS1. Figure 1B shows these genes with the fold change identified in our analyses (GDS3592) and the confirmation performed using data contained at Oncomine (www.oncomine.org). All genes included within the cell cycle function are described in Supplementary table 1.

Before evaluating compounds against these kinases, it was relevant to know if molecular alterations of these genes exist in ovarian tumors. To do so we used data from 311 tumors contained at cBioportal [18] observing that AURKA was amplified in $8.7 \%$ of tumors and CHEK1 in 3,9\%. TTK/MPS1 was amplified in $1.6 \%$ and AURKB was not amplified (Figure $1 \mathrm{C}$ ).

Next, we analyzed the anti-proliferative capacity of agents against the proteins coded for those genes that were amplified (AURKA, CHEK1 and TTK/MPS1) in a panel of four ovarian cell lines including OVCAR3, OVCAR8, IGROV1 and 
SKOV3. To assess the effect on proliferation of new drugs targeting these kinases we used three compounds: LY2603618 against the CHEK1 [20, 21]; Alisertib (MLN8237) against AURKA [22, 23]; and AZ3146 against TTK/MPS1 $[24,25]$. The most anti-proliferative effect was observed for LY2603618 and Alisertib, targeting AURKA and CHEK1 (Figure 1D and E). We observed less activity for AZ3146 (Figure 1F). To evaluate the therapeutic index of LY2603618 and Alisertib we used the non-transformed cell line model HACAT, observing that the doses required to produce anti-proliferative effect were higher than in tumoral ones (Supplementary Figure 1).

\section{Targeting of Aurora Kinases A synergizes with CHEK1 inhibition}

Next, we decided to combine inhibitors of CHEK1 and AURKA together as were the most active compounds in our cellular screening. Combination of LY2603618 with Alisertib was synergistic at most of the doses tested (Figure 2A). Increasing doses of LY2603618 augmented the effect of a fixed dose of Alisertib in OVCAR8 and OVCAR3 (Figure 2B). Studies using semi-solid media with matrigel, showed a similar effect with the combination, being the effect more evident in OVCAR3 (Figure 2C). We also explored the effect of the combination using clonogenic assays showing similar results (Supplementary Figure 2A). Finally, we confirmed the cytotoxic effect by performing a LDH assay of the combination compared with each agent alone in OVCAR3 and OVCAR8 (Supplementary Figure 2B).

Combination of both compounds induces cell cycle arrest leading to apoptosis 
Given the fact that the combination of both compounds inhibited cell proliferation when evaluated in different models; we decided to explore if this effect was due to a cell cycle arrest or an induction of apoptosis. To do this, we used the two most sensitive cell lines, OVCAR3 and OVCAR8. Treatment with Alisertib showed a profound arrest at the G2/M phase, while treatment with LY2603618 showed a slight increase at G1 that was more evident in OVCAR8 (Figure $3 \mathrm{~A}$ ). Treatment with the combination produced an arrest at $\mathrm{G} 2 / \mathrm{M}$ in OVCAR8; effect that was less evident in OVCAR3 and SKOV3 (Figure 3A and Supplementary Figure 3). The biochemical evaluation of G2/M components showed an increase in pH3 in OVCAR8 at 12 hours, indicative of arrest in $\mathrm{M}$ (Figure 3B). This effect was less seen in OVCAR3. No clear modifications of p21 and p27 were observed with the combination (Supplementary Figure 3).

To confirm the effect of Alisertib and LY2603618 on Aurora Kinases and CHEK1, we performed western blot studies with these compounds given alone or in combination at 12 and 24 hours. LY2603618 was able to inhibit the phosphorylation of CHEK1 and Alisertib did not affect other Aurora Kinase isoforms like AURKB or AURKC. Treatment with nocodazole arrested cells at G2 (Figure 3C).

To analyzed the effect of the combination on the mitotic process we evaluated the percentage of aberrant mitotic spindles. Combination of LY2603618 and Alisertib showed an increase in the formation of aberrant spindles that was more profound in OVCAR8, in line with the arrest observed at G2/M and the increase in $\mathrm{pH} 3$ (Figure 3D). Supplementary figure 4A shows random images in OVCAR3 and OVCAR8 of aberrant mitotic spindles for each treatment. 
Next, we evaluated the effect of this combination on cell death. As can be seen in figure $4 \mathrm{~A}$, administration of the combination induced apoptosis in both OVCAR3 and OVCAR8. Treatment with the pan-caspase inhibitor Z-VAD-FMK partially reduced the induction of apoptosis, demonstrating that some of the apoptosis induction was mediated by caspases (Figure 4B). This observation was further confirmed by the evaluation of caspase 3 activity in OVCAR3 and OVCAR8 (Supplementary Figure 4B) The biochemical analysis demonstrated that the combination increased $\mathrm{pH} 2 \mathrm{AX}$ in both cell lines, a marker of DNA damage and did not induce PARP degradation (Figure 4C).

The combination of Alisertib and LY2603618 affects stem cell like properties

As stem-cells play a major role in ovarian relapse, we decided to explore the effect of the combination on stem cell properties. We first explored expression of the stem cell biomarkers CD44 and CD133 in OVCAR3 and OVCAR8 after treatment with Alisertib, LY2603618 and the combination in the overall population of adherent cells. We observed that combination slightly reduced surface expression of CD44 and CD133 when compared with each agent given alone (Figure 5A and Supplementary Figure 5). As cells grown as tumorspheres (TS) better recapitulate stem cell properties [26], we evaluated the effect of each drug alone or the combination on secondary TS formation. Figure 5B shows enrichment in the stem cell markers CD44 and SOX2 on OVCAR3 and OVCAR8-derived TS, confirming that derived TS are an appropriate model to evaluate the effect of drugs. The combination was able to reduce the formation of TS in a greater manner than when each agent was given alone (Figure $5 \mathrm{C}$ ). Finally, we evaluated the effect on tumorspheres of both agents given alone or 
in combination with chemotherapy. As can be seen in supplementary figure 6, the combination increased the effect of individual treatments.

The combined inhibition synergizes with chemotherapies

To translate our findings to the clinical setting we evaluated the synergistic interaction of LY2603618 and Alisertib alone or when given with docetaxel and carboplatin. We decided to use these chemotherapies as platinum-based agents and taxanes are the main treatment in ovarian cancer. The combination augmented the effect of each agent given alone (Figure 6A), and showed a synergistic interaction for most of the doses used in three cell lines, SKOV3, OVCAR3 and OVCAR8 (Supplementary Figure 1). These data demonstrate that the administration of LY2603618 and Alisertib could have a potential translation to patients if given with standard chemotherapy.

\section{Association of Aurora Kinase A and CHEK1 gene expression with outcome}

Finally, we decided to evaluate the association of these kinases with clinical outcome using public transcriptomic data [19]. The concomitant expression of Aurora Kinase A and CHEK1 was linked to detrimental progression free survival (PFS) and overall survival (OS) in early stage ovarian cancer patients (Figure $6 \mathrm{~B})$. These data confirmed the implication of these kinases in the oncogenic phenotype of ovarian tumors.

\section{Discussion}

In the present article we describe a synergistic antitumoral effect between AURKA and CHEK1 inhibitors in ovarian cancer. We used an in silico analysis 
to identify relevant functions that can be pharmaceutically inhibited. These studies permitted the identification of some kinases that regulate cell cycle progression. Of note, the upregulated genes identified in this small dataset were confirmed with data contained at Oncomine, a database that includes a large number of patients. Moreover, some of these genes participate in pathways that are involved in the pathophysiology of this tumor [11]. By using public data from TCGA, we identified that AURKA and CHEK1 were amplified in $8.7 \%$ and $3.9 \%$ of ovarian tumors, respectively; providing the rational for exploring agents against these kinases. Although our in silico approach is valid, confirmation of our results using data from a prospective cohort of patients would reinforced the findings.

Next, we aimed to explore agents against the identified proteins. Interestingly Alisertib, a kinase inhibitor against AURKA, and LY2603618 a CHEK1 inhibitor, were the most active compounds compared with the TTK/MPS1 inhibitor AZ3146. It is relevant to mention that some Aurora Kinases, as well as other FOXM1-controlled cell cycle proteins such as CDC25, cyclin B and PLK1, have been reported to be overexpressed in ovarian cancer [11]. In addition, among the combination of agents used, AURKA inhibition synergized with CHEK1 inhibitors, and this was not observed when combining other agents. Alisertib is an AURKA inhibitor that is currently at its late stage in clinical development in non-small cell lung cancer (NSCLC) and has shown activity in several solid tumors [12, 13]. CHEK1 inhibitors including LY2603618 are in early stage clinical development in different tumor types, mainly NSCLC [21].

When evaluating the mechanism of action, Alisertib induced arrest at the G2/M phase as it is an agent that affects kinases involved in the formation of the 
mitotic spindle. Inhibition of CHEK1 with LY2603618 induced arrest at G1. Combination of both agents increased G2/M arrest at different levels, and produced a profound induction of apoptosis. This finding is of remarkable interest as each agent alone showed a cytostatic effect, but the combination was cytotoxic, a desirable effect when treating cancer patients.

As most of cancer deaths are associated with tumor relapses and tumor cells with stem cell properties are involved in this process, we decided to explore the effects of the combination on the stem cell population. The combination of both compounds reduced the expression of stem cell biomarkers in a greater magnitude than each agent alone. Moreover, self-renewal capability was also altered, as observed when evaluating secondary TS formation after treatment. Some studies have described the effect of the individual inhibition of these kinases on the stem cell properties $[27,28]$. However, this is the first time that this effect has been described with the combination.

Translating preclinical drug combinations to potential uses in the clinical setting is a main goal. To do so we explored the activity of this combination with standard chemotherapy used in ovarian cancer, including docetaxel and carboplatin. OVCAR3 and OVCAR8 showed different sensitivity to docetaxel and carboplatin, but administration of LY2603618 and Alisertib increased the activity of each agent given alone. Globally, these results open the possibility to further explore these combinations in the clinical setting.

Finally, we identified that expression of AURKA and CHEK1 was associated with detrimental outcome in early stage ovarian cancer. These findings, together with the genomic studies that reported deregulation of cell cycle and DNA repair pathways in ovarian cancer, have two important implications. First, the available 
data demonstrate the oncogenic activity of these kinases in ovarian cancer. Secondly, molecular analyses of the pathways in which these genes participate may be used to select patients sensitive to these agents. Of note, administration

of targeted agents against amplified genes has shown clinical utility as is the case for HER2 in breast cancer [29].

In conclusion, we describe a novel combination of agents for the treatment of ovarian cancer with potential implications in the clinical setting. Amplification of AURKA and CHEK1 is observed in more than $12 \%$ of the cases, opening the possibility to develop this combination in patients with amplifications of these genes.

\section{Acknoledgements}

We would like to thanks G. Serrano for her technical support.

I also thank my parents Pilar Fernández and Santiago Ocana for his support during my professional life. 


\section{References}

1. Ocana A, Pandiella A. Personalized therapies in the cancer "omics" era. Mol Cancer 2010; 9: 202.

2. Ocana A, Pandiella A, Siu LL, Tannock IF. Preclinical development of molecular-targeted agents for cancer. Nat Rev Clin Oncol 2010; 8: 200-209.

3. Pagliarini R, Shao W, Sellers WR. Oncogene addiction: pathways of therapeutic response, resistance, and road maps toward a cure. EMBO Rep 2015; 16: 280-296.

4. Bookman MA. Optimal primary therapy of ovarian cancer. Ann Oncol 2016; 27 Suppl 1: i58-i62.

5. Jackson AL, Eisenhauer EL, Herzog TJ. Emerging therapies: angiogenesis inhibitors for ovarian cancer. Expert Opin Emerg Drugs 2015; 20: 331-346.

6. Ledermann JA, El-Khouly F. PARP inhibitors in ovarian cancer: Clinical evidence for informed treatment decisions. Br J Cancer 2015; 113 Suppl 1: S1016.

7. Montero JC, Chen X, Ocana A, Pandiella A. Predominance of mTORC1 over mTORC2 in the regulation of proliferation of ovarian cancer cells: therapeutic implications. Mol Cancer Ther 2012; 11: 1342-1352.

8. Montero JC, Garcia-Alonso S, Ocana A, Pandiella A. Identification of therapeutic targets in ovarian cancer through active tyrosine kinase profiling. Oncotarget 2015; 6: 30057-30071.

9. Ocana A, Perez-Pena J, Alcaraz-Sanabria A et al. In silico analyses identify gene-sets, associated with clinical outcome in ovarian cancer: role of mitotic kinases. Oncotarget 2016; 7: 22865-22872.

10. Dominguez-Brauer $\mathrm{C}$, Thu $\mathrm{KL}$, Mason JM et al. Targeting Mitosis in Cancer: Emerging Strategies. Mol Cell 2015; 60: 524-536.

11. Cancer Genome Atlas Research N. Integrated genomic analyses of ovarian carcinoma. Nature 2011; 474: 609-615.

12. Kozyreva VK, Kiseleva AA, Ice RJ et al. Combination of Eribulin and Aurora A Inhibitor MLN8237 Prevents Metastatic Colonization and Induces Cytotoxic Autophagy in Breast Cancer. Mol Cancer Ther 2016; 15: 1809-1822. 
13. Pitts TM, Bradshaw-Pierce EL, Bagby SM et al. Antitumor activity of the aurora a selective kinase inhibitor, alisertib, against preclinical models of colorectal cancer. Oncotarget 2016; 7: 50290-50301.

14. Bast RC, Jr., Hennessy B, Mills GB. The biology of ovarian cancer: new opportunities for translation. Nat Rev Cancer 2009; 9: 415-428.

15. Zhao $\mathrm{H}$, Watkins $\mathrm{JL}$, Piwnica-Worms $\mathrm{H}$. Disruption of the checkpoint kinase 1/cell division cycle 25A pathway abrogates ionizing radiation-induced $S$ and G2 checkpoints. Proc Natl Acad Sci U S A 2002; 99: 14795-14800.

16. Dutertre S, Cazales M, Quaranta M et al. Phosphorylation of CDC25B by Aurora-A at the centrosome contributes to the G2-M transition. J Cell Sci 2004; 117: 2523-2531.

17. Roshak AK, Capper EA, Imburgia $C$ et al. The human polo-like kinase, PLK, regulates cdc2/cyclin $B$ through phosphorylation and activation of the cdc25C phosphatase. Cell Signal 2000; 12: 405-411.

18. Gao J, Aksoy BA, Dogrusoz $U$ et al. Integrative analysis of complex cancer genomics and clinical profiles using the cBioPortal. Sci Signal 2013; 6: pl1.

19. Gyorffy B, Lanczky A, Szallasi Z. Implementing an online tool for genome-wide validation of survival-associated biomarkers in ovarian-cancer using microarray data from 1287 patients. Endocr Relat Cancer 2012; 19: 197208.

20. Matthews TP, Jones AM, Collins I. Structure-based design, discovery and development of checkpoint kinase inhibitors as potential anticancer therapies. Expert Opin Drug Discov 2013; 8: 621-640.

21. King C, Diaz H, Barnard D et al. Characterization and preclinical development of LY2603618: a selective and potent Chk1 inhibitor. Invest New Drugs 2014; 32: 213-226.

22. Sells TB, Chau R, Ecsedy JA et al. MLN8054 and Alisertib (MLN8237): Discovery of Selective Oral Aurora A Inhibitors. ACS Med Chem Lett 2015; 6: 630-634.

23. Niu H, Manfredi M, Ecsedy JA. Scientific Rationale Supporting the Clinical Development Strategy for the Investigational Aurora A Kinase Inhibitor Alisertib in Cancer. Front Oncol 2015; 5: 189. 
24. Vijay Kumar D, Hoarau C, Bursavich M et al. Lead optimization of purine based orally bioavailable Mps1 (TTK) inhibitors. Bioorg Med Chem Lett 2012; 22: 4377-4385.

25. Tipton AR, Ji W, Sturt-Gillespie B et al. Monopolar spindle 1 (MPS1) kinase promotes production of closed MAD2 (C-MAD2) conformer and assembly of the mitotic checkpoint complex. J Biol Chem 2013; 288: 3514935158.

26. Weiswald LB, Bellet D, Dangles-Marie V. Spherical cancer models in tumor biology. Neoplasia 2015; 17: 1-15.

27. Wang WJ, Wu SP, Liu JB et al. MYC regulation of CHK1 and CHK2 promotes radioresistance in a stem cell-like population of nasopharyngeal carcinoma cells. Cancer Res 2013; 73: 1219-1231.

28. Zheng FM, Long ZJ, Hou ZJ et al. A novel small molecule aurora kinase inhibitor attenuates breast tumor-initiating cells and overcomes drug resistance. Mol Cancer Ther 2014; 13: 1991-2003.

29. Esparis-Ogando A, Montero JC, Arribas $\mathrm{J}$ et al. Targeting the EGF/HER Ligand-Receptor System in Cancer. Curr Pharm Des 2016; 22: 5887-5898. 


\section{Figure Legends}

Figure 1. Identification of druggable cell cycle kinases in ovarian cancer

A. Gene expression analysis comparing non-transformed human epithelial ovarian cells and ovarian carcinoma cells using data contained at GDS3592. Functional annotation of deregulated genes as reported by DAVID Bioinformatics 6.8. B. Fold change and p-value of Aurora Kinase A, Aurora Kinase B, Checkpoint kinase 1 and TTK protein Kinase/MPS1 from data contained at GDS3592 and Oncomine (www.oncomine.org). C. Gene alterations (amplifications, deletions and mutations) in AURKA, AURKB, CHEK1 and TTK/MPS1 were studied using cBioportal. D, E, F. Effect of Aurora kinase A inhibitor (Alisertib) (D), CHEK1 inhibitor (LY2603618) (E) and TTK protein kinase/MPS1 inhibitor (AZ3146) (F) on cell proliferation in OVCAR3, OVCAR8, SKOV3 and IGROV1 using MTT assays, as described in material and methods. Student t-test was used to determine statistical significance between control and the most effective concentrations.

Figure 2. Synergistic effect of Aurora kinases and Chk1 inhibitors in OVCAR3 and OVCAR8 cell lines

A, B. Cells were treated with the indicated doses of LY2603618 and Alisertib during $72 \mathrm{~h}$. Then, metabolization of MTT in viable cells was determined by spectrophotometry. Synergistic effects were analyzed with CalcuSyn program (A). Percentage of viable cells is represented as MTT metabolization (B). C. 3D culture experiments on OVCAR3 and OVCAR8. Cells were seeded on matrigelcoated wells and, $24 \mathrm{~h}$ later, treated with the indicated doses (IC50 values) of Alisertib, LY2603618 or the combination of both drugs for $72 \mathrm{~h}$. 3D colonies formation was evaluated by microscopy. Percentage of 3D colonies referred to 
control (left panel) and contrast phase images (right panel) are shown. Student t-test was used to determine statistical differences $p \leq 0.05^{*}, p \leq 0.01^{* *}$, $p \leq 0.001^{* * *}(B, C)$

Figure 3. Alisertib and LY2603618 induce cell cycle arrest and formation of aberrant mitotic spindles in ovarian cancer cells

A. OVCAR3 and OVCAR8 cells were treated with Alisertib, LY2603618 and the combination of both drugs at the indicated doses. $24 \mathrm{~h}$ later, cell cycle progression was evaluated by flow cytometry. B. Expression of cell cyclerelated proteins, Cyclin $\mathrm{B}$, Wee1, $\mathrm{pCDK} 1$ and $\mathrm{pH} 3$, was measured by western blot at 12 and $24 \mathrm{~h}$ after treatment with Alisertib, LY2603618 and the combination at the indicated doses. C. Proteins expression of Aurora A, pAurora $(A, B, C)$, CHEK1 and pCHEK1 in OVCAR3 and OVCAR8 treated with Alisertib, LY2603618 and both drugs at 12 and 24 h D. Aberrant mitotic spindles formation was determined by immunofluorescence microscopy after $24 \mathrm{~h}$, after treatment at the indicated doses. Figure represents the percentage of aberrant mitotic spindles. Student t-test differences $p \leq 0.05^{*}, p \leq 0.01^{* *}, p \leq 0.001^{* * *}$.

Figure 4. Alisertib and LY2603618 induce caspase-dependent death in ovarian cells

A. OVCAR3 and OVCAR8 cells were treated with the indicated doses of Alisertib, LY2603618 or both drugs in combination for $72 \mathrm{~h}$. Then, percentage of Annexin $\mathrm{V}+/$ - cells was determined by flow cytometry. B. Cells were pre-treated with the pan-caspase inhibitor Z-VAD $(50 \mu \mathrm{M})$ for $1 \mathrm{~h}$ before being exposed to the drugs. Then, percentage of apoptotic cells was analyzed by flow cytometry at 72 h. C. After drug exposure for the indicated times, PARP and $\mathrm{pH} 2 \mathrm{AX}$ expression was evaluated by western blot as described in material and 
methods. GAPDH was used as a loading control. Student t-test differences $p \leq 0.05^{*}, p \leq 0.01^{* *}, p \leq 0.001^{* * *}$

Figure 5. Combination of Alisertib and LY2603618 decreases stemness capability in ovarian cancer cells

A. OVCAR3 and OVCAR8 cells were treated as indicated, and $72 \mathrm{~h}$ later cells were collected and surface expression of CD44 and CD133 was determined by flow cytometry. B. CD44 and Sox-2 expression on OVCAR3 and OVCAR8derived tumorspheres (TS) was evaluated by inmunofluorence using confocal microscopy, as described in material and methods. C. Secondary formation assays were performed on OVCAR3 and OVCAR8-derived TS to evaluate the effect of Alisertib, LY2603618 and the combination on self-renewal capability. Results are represented as number of TS per fields of view (FOV) (left panel). Representative contrast phase photographs at $72 \mathrm{~h}$ are also shown (right panel). Student t-test differences $p \leq 0.05^{*}, p \leq 0.01^{* *}, p \leq 0.001^{* * *}$.

Figure 6. AURKA/B and CHK1 overexpression correlates with worse prognosis in ovarian cancer patients

A. OVCAR3 and OVCAR8 were treated with Alisertib and LY2603618 alone or in combination with docetaxel (left panel) and carboplatin (right panel) at the indicated doses. Metabolization of MTT in viable cells was determined by spectrophotometry as described in material and methods. Two-ways Student's test differences $p \leq 0.05^{*}, p \leq 0.01^{* *}, p \leq 0.001^{* * *}$. B. Kaplan-Meier curves for progression free (PFS) and overall survival (OS) for AURKA/CHEK1 expression using the KM plotter online tool, as described in material and methods. 
A

NON-TRANSFORMED

HUMAN EPITHELIAL

OVARIAN CELLS

$\mathrm{n}=12$

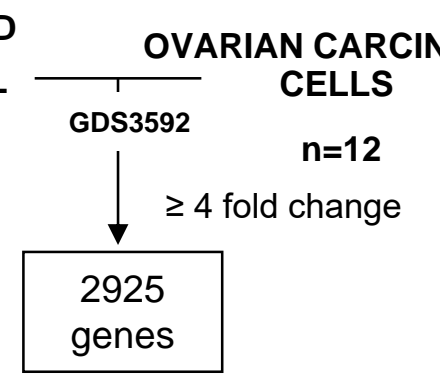

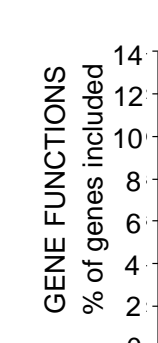

B

\begin{tabular}{|c|c|c|c|c|c|}
\hline ID GENE & DESCRIPTION & $\begin{array}{c}\text { FOLD } \\
\text { CHANGE } \\
\text { GDS3592 }\end{array}$ & $\begin{array}{c}\text { ANOVA } \\
\text { P-VALUE } \\
\text { GDS3592 }\end{array}$ & $\begin{array}{c}\text { FOLD } \\
\text { CHANGE } \\
\text { ONCOMINE }\end{array}$ & $\begin{array}{c}\text { P-VALUE } \\
\text { ONCOMINE }\end{array}$ \\
\hline 208079_s_at & Aurora kinase A & 7 & $3,64 \mathrm{E}-08$ & 6.504 & $6.53 \mathrm{E}-8$ \\
\hline 239219_at & Aurora kinase B & 5.1 & 0,001028 & 2.818 & $2.34 \mathrm{E}-5$ \\
\hline 205394_at & Checkpoint kinase 1 & 4.6 & 0,000004 & 4.147 & $2.43 \mathrm{E}-7$ \\
\hline 204822_at & $\begin{array}{c}\text { TTK/MPS1 protein } \\
\text { kinase }\end{array}$ & 7.21 & $7,51 \mathrm{E}-07$ & 15.153 & $2.06 \mathrm{E}-9$ \\
\hline
\end{tabular}

C

Figure 1

\begin{tabular}{|c|c|c|c|}
\hline \multicolumn{4}{|c|}{311 Ovarian Serous Cystadenocarcinome Samples } \\
\hline GENE NAME & Amplification & Deletion & Mutation \\
\hline Aurora kinase A & $8.70 \%$ & - & - \\
\hline Aurora kinase B & $0.60 \%$ & $0.60 \%$ & - \\
\hline Checkpoint kinase 1 & $3.90 \%$ & $0.60 \%$ & - \\
\hline $\begin{array}{c}\text { TTK/MPS1 protein } \\
\text { kinase }\end{array}$ & $1.6 \%$ & $0.3 \%$ & $0.6 \%$ \\
\hline
\end{tabular}

E

LY2603618 ( $\mu \mathrm{M})$

F

AZ3146 ( $\mu \mathrm{M})$
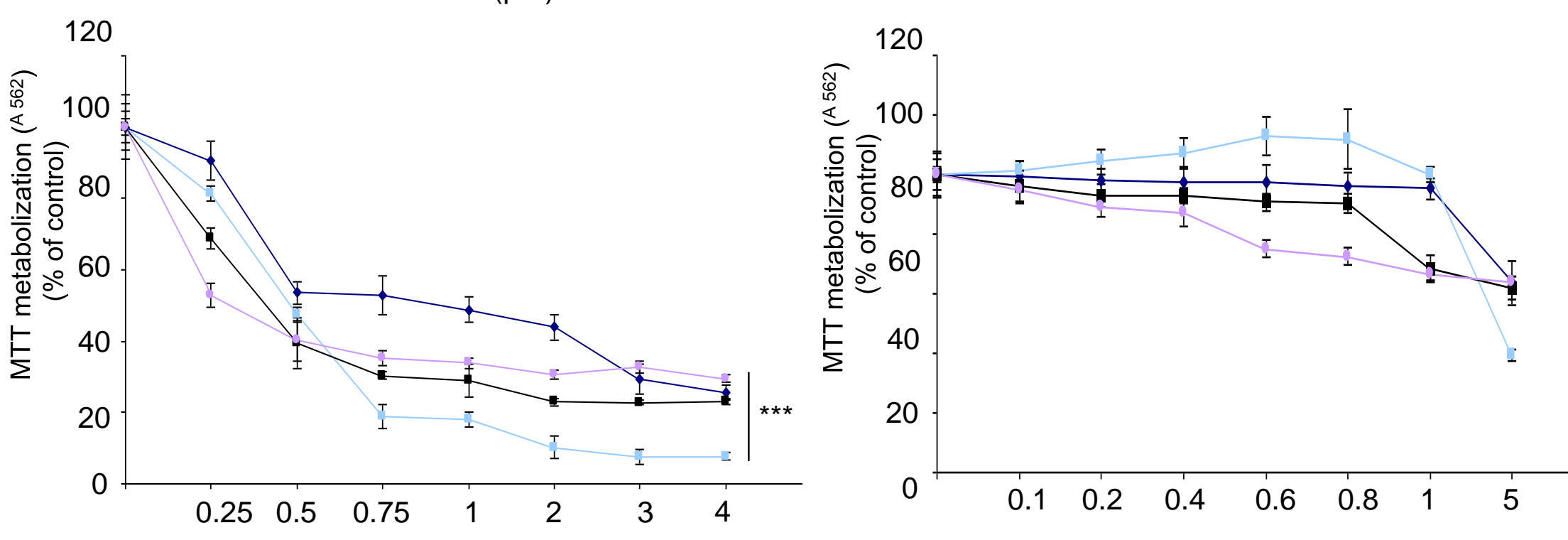

Downloaded from mct.aacrjournals.org on August 17, 2018. @ 2017 American Association for Cancer Research. 
A

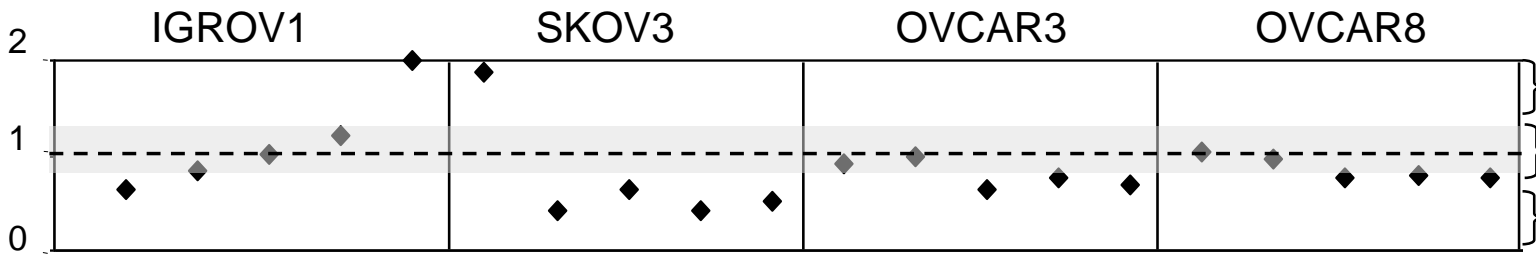

Figure 2

LY2603618(nM) $50100150 \quad 200 \quad 250 \quad 50 \quad 100 \quad 150 \quad 200300200300400 \quad 500600200300400500600$

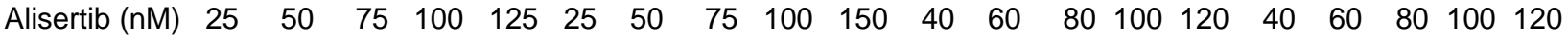
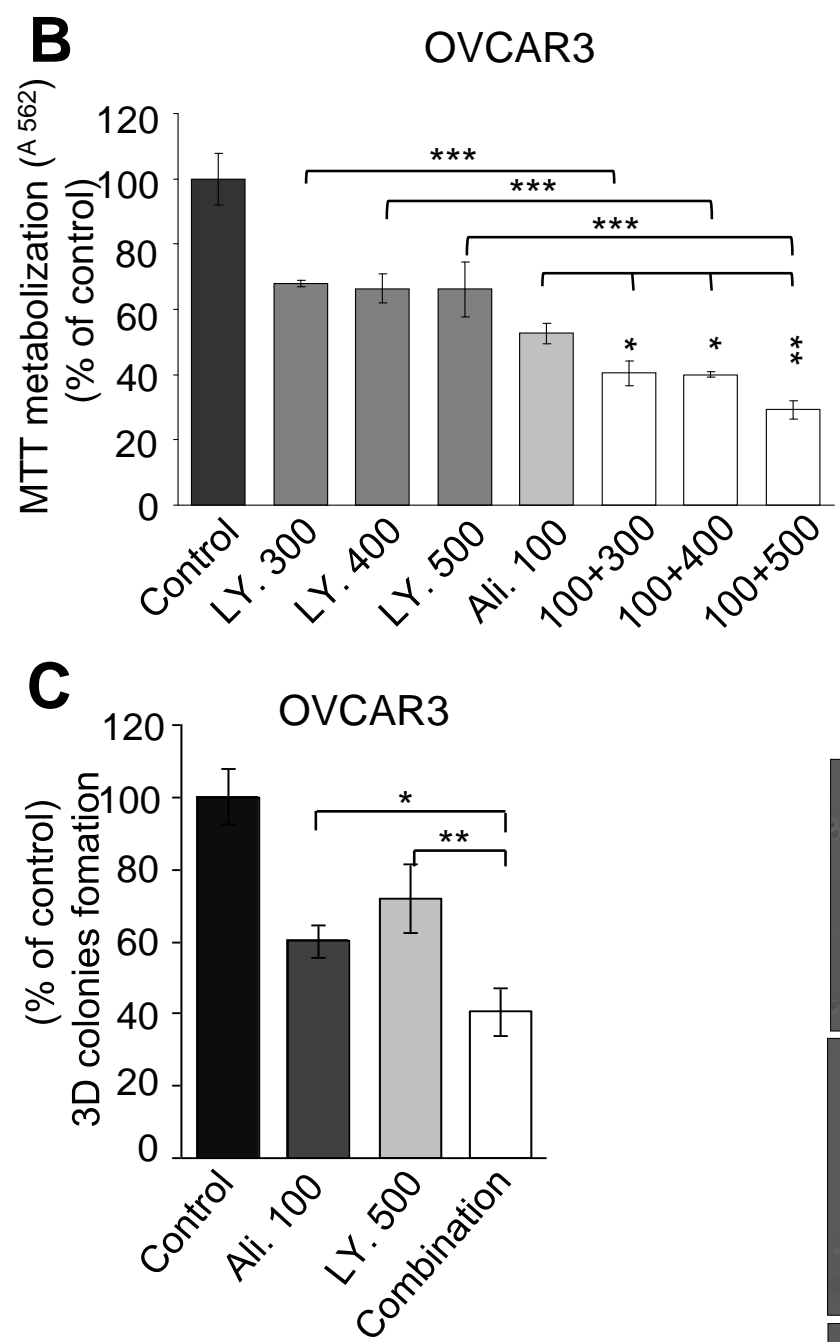

\section{OVCAR8}

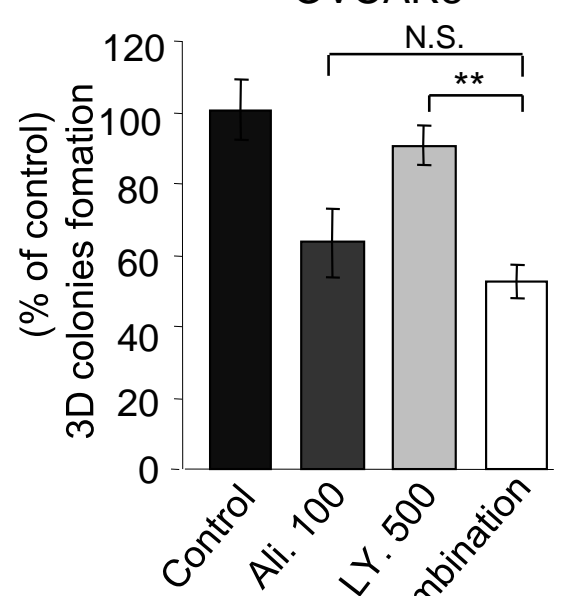

OVCAR8

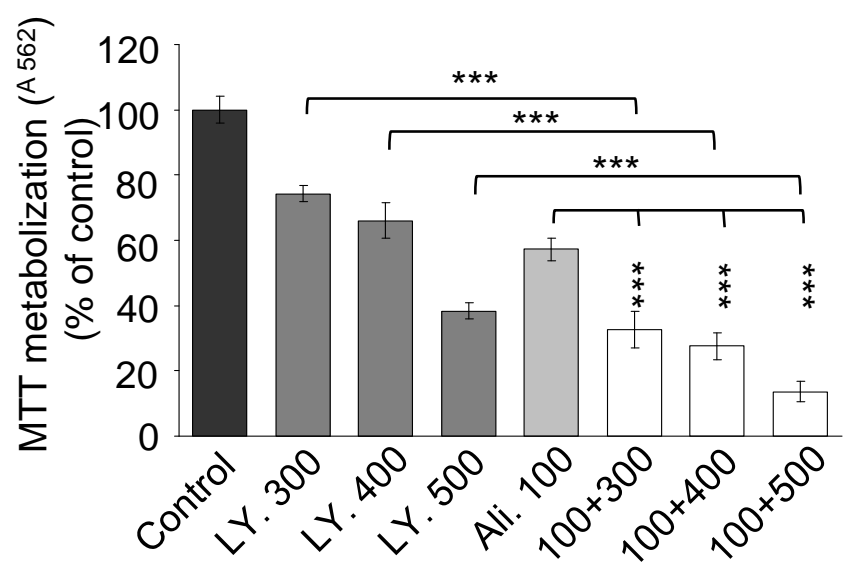

OVCAR3 OVCAR8

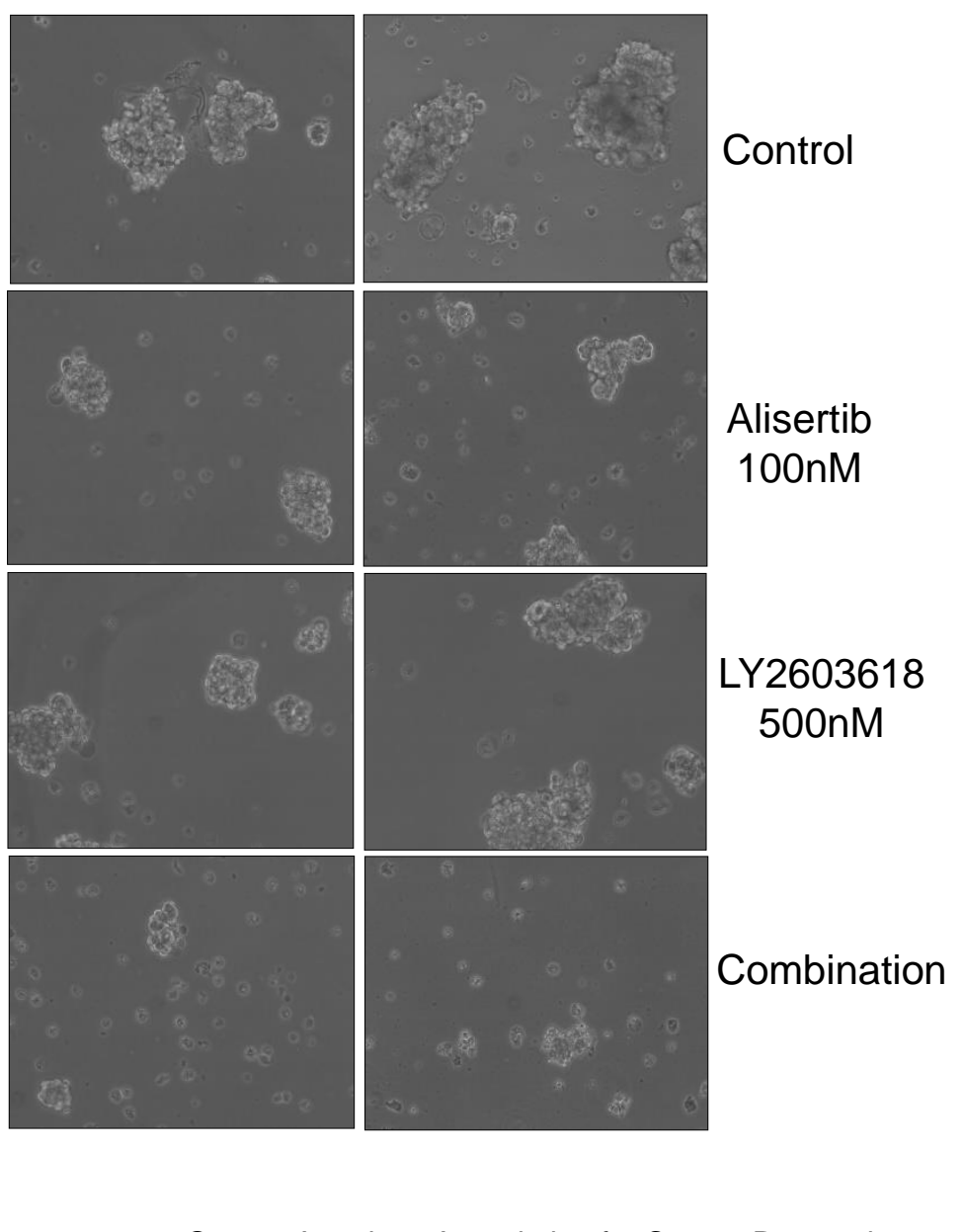

Downloaded frowe 
A

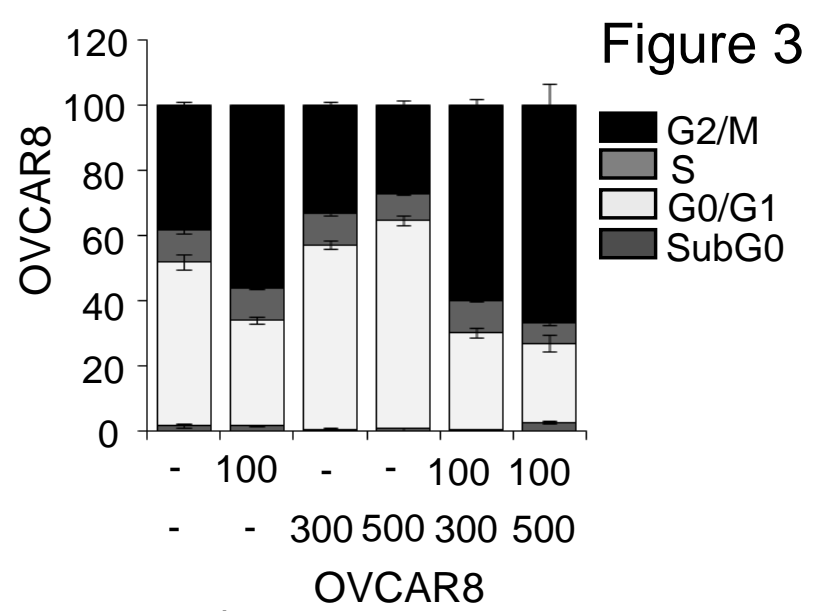

B

LY2603618 (nM) - - 300500300500

\section{OVCAR8}

12 hours 24 hours

Alisertib (nM) $-100-100-100-100$ LY2603618(nM) - $\quad-500500 \quad-\quad-500500$

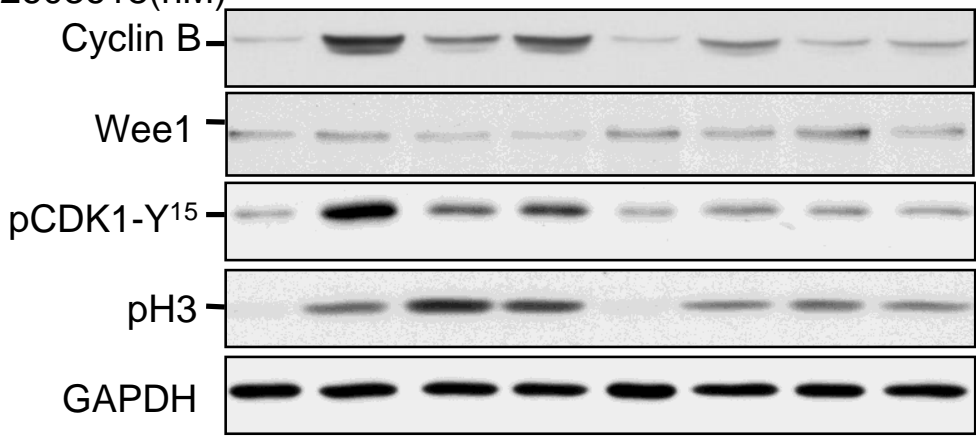

C

OVCAR3

BT474

OVCAR8

12 hours 24 hours Nocodazole 12 hours 24 hours Nocodazole

Alisertib $-100-100-100-100 \mid-100-100-100-100$ LY2603618 - $\quad-500500-\quad-500500$ จ

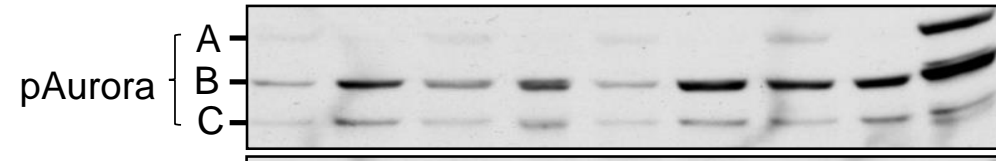
pAurora $A-$

Aurora A pChk1

Chk1

GAPDH
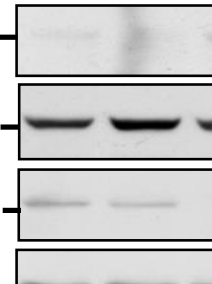


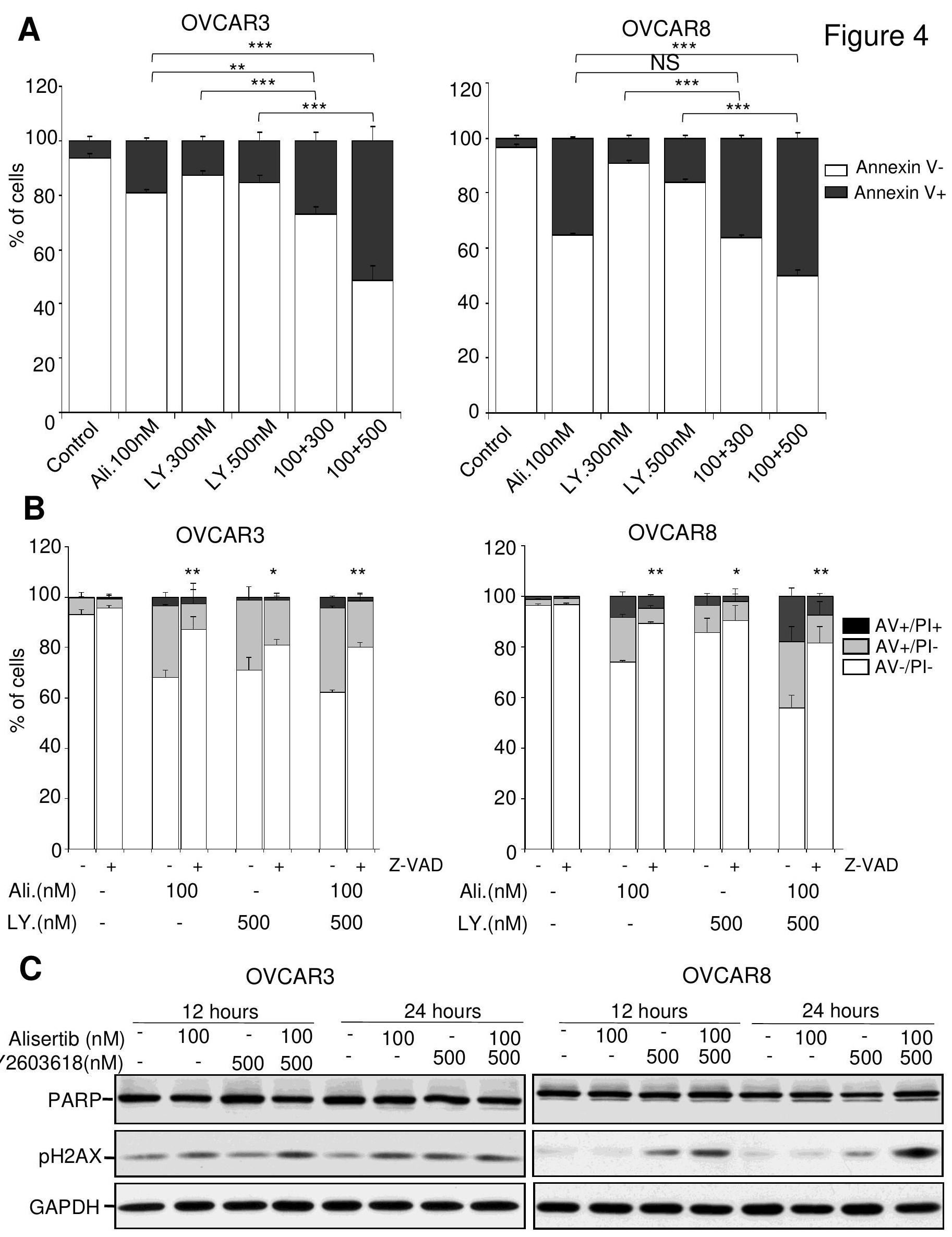



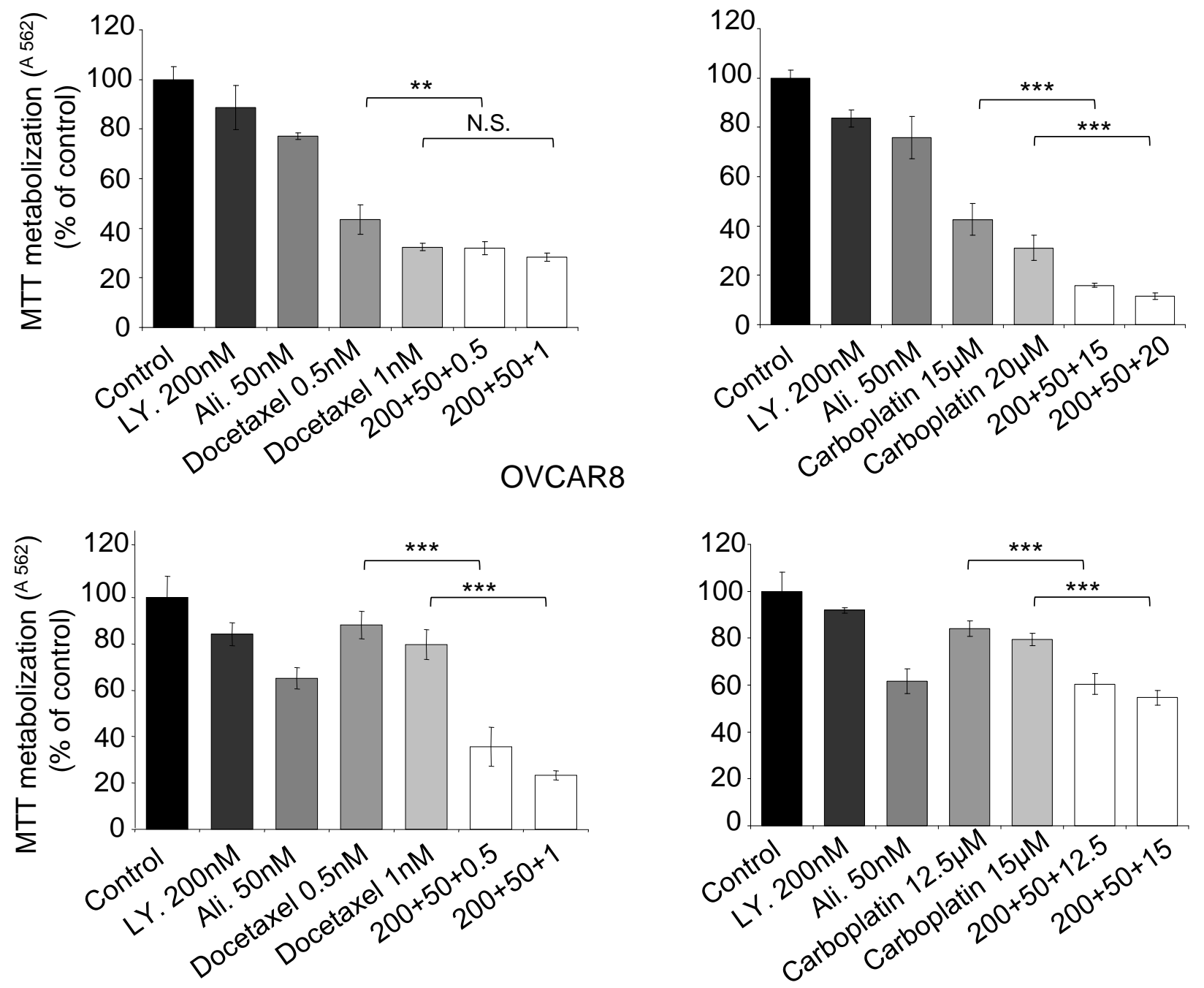

B

PFS

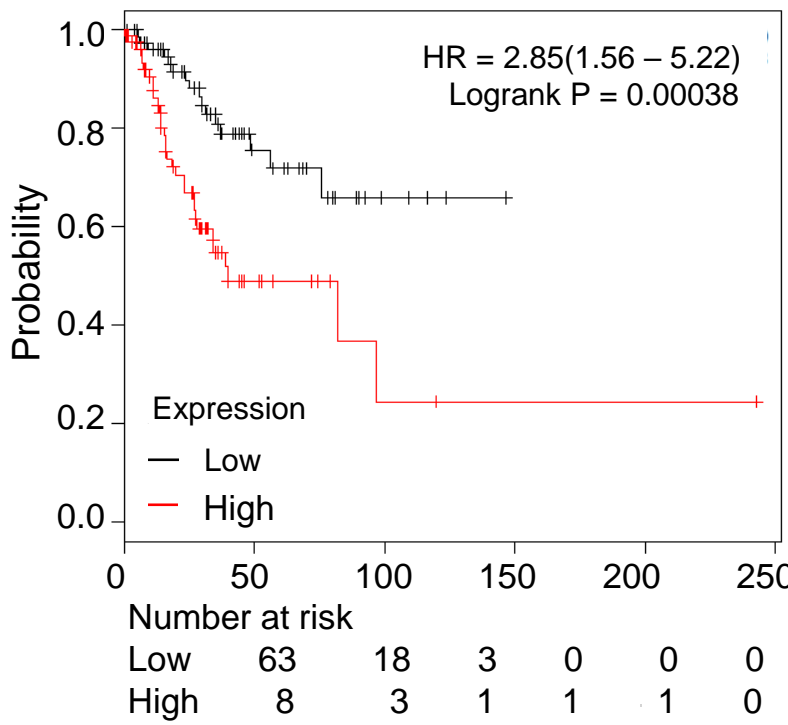

AURKA/CHEK1

OS

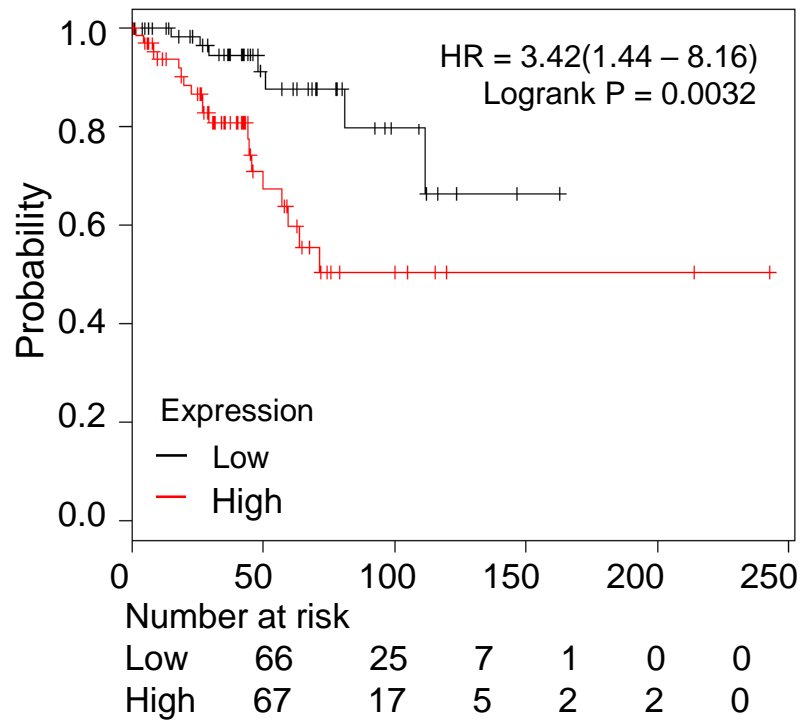




\section{Molecular Cancer Therapeutics}

\section{Synthetic lethality interaction between Aurora kinases and CHEK1 inhibitors in ovarian cancer}

Ana Alcaraz-Sanabria, Cristina Nieto-Jiménez, Verónica Corrales-Sánchez, et al.

Mol Cancer Ther Published OnlineFirst August 28, 2017.

Updated version Access the most recent version of this article at: doi:10.1158/1535-7163.MCT-17-0223

Supplementary Access the most recent supplemental material at:

Material http://mct.aacrjournals.org/content/suppl/2017/08/26/1535-7163.MCT-17-0223.DC1

Author Author manuscripts have been peer reviewed and accepted for publication but have not yet Manuscript been edited.

E-mail alerts Sign up to receive free email-alerts related to this article or journal.

Reprints and Subscriptions

Permissions
To order reprints of this article or to subscribe to the journal, contact the AACR Publications Department at pubs@aacr.org.

To request permission to re-use all or part of this article, use this link http://mct.aacrjournals.org/content/early/2017/08/26/1535-7163.MCT-17-0223.

Click on "Request Permissions" which will take you to the Copyright Clearance Center's (CCC) Rightslink site. 